\title{
Distribución de cangrejos ermitaños (Anomura: Paguroidea) en el mar Caribe colombiano
}

\author{
Bibian Martínez Campos ${ }^{1}$, Néstor Hernando Campos $^{2}$ \& Adriana Bermúdez Tobón ${ }^{3}$ \\ 1. Programa de Biología Marina, Facultad de Ciencias Naturales, Universidad Jorge Tadeo Lozano Santa Marta; \\ usagiblacklady@gmail.com \\ 2. CECIMAR, Universidad Nacional de Colombia, Sede Caribe, en INVEMAR, Santa Marta; nhcamposc@unal.edu.co \\ 3. Facultad de Ciencias Exactas y Naturales. Universidad de Cartagena; abermudezt@ unicartagena.edu.co
}

\section{Recibido 01-III-2011. Corregido 15-VIII-2011. Aceptado 12-IX-2011.}

\begin{abstract}
Hermit crabs (Anomura: Paguroidea) distribution patterns in the Colombian Caribbean Sea. Hermit crabs represent the marine life in the Colombian Caribbean, and are important for the dynamic equilibrium maintenance in ecosystems, the ecological interactions and their impact on food web stability. Generally, in order to come up with some conservation strategies, strong bio-geographical information is needed for policies definition. With this aim, this study analyzed the distribution patterns of hermit crabs in the Colombian Caribbean Sea, through classification and spatial ordination multivariate analyses, using historical records from years 1916 to 2006. Besides, the world distribution of Colombian species and their geographic affinity in the Caribbean and Western Atlantic were identified. The results show deep differences between coastal and continental slope faunas, and latitudinal differences in the assemblages, with the identification of three groups: Northeast, Center and Southwest. The differences in faunal composition that support these three groups were determined. Based on maps of the Colombian marine ecosystems, it was found that the main factors affecting the distribution of hermit crabs were the Caribaná slope (depth), water-mass temperature, Guajira sea-grass beds, and particular conditions of "Coralline Archipelagos" and "Darién" eco-regions. Colombian hermit crab fauna is more related to the North Atlantic and the Antilles, than to the South Atlantic and the Gulf of Mexico. Additionally, geographical sub-provinces in which Colombia is included, these were found as transition zones among Northern and Austral subprovinces of the Greater Caribbean. Rev. Biol. Trop. 60 (1): 233-252. Epub 2012 March 01.
\end{abstract}

Key words: hermit crabs, distribution patterns, multivariate analysis, Colombian Caribbean, latitudinal distribution, depth distribution.

Conocer los recursos biológicos y la forma en la que se distribuyen permite implementar estrategias de conservación eficientes no sólo para las especies, sino además para las biocenosis que éstas integran; esta responsabilidad es aún mayor en un país "megadiverso" como Colombia, cuyo mar territorial de $980000 \mathrm{~km}^{2}$, con costas sobre el Océano Pacífico y el Mar Caribe, alberga aproximadamente el $10 \%$ de la biodiversidad de los océanos del mundo (Díaz \& Gómez-López 2000, Ardila etal. 2002).

El estudio de la distribución y el estado de las comunidades es muy importante en los cangrejos ermitaños, ya que al carecer de interés comercial, han sido poco estudiados a pesar que derempeñan un papel importante en el equilibrio dinámico de los ecosistemas, dada su diversidad, impacto en la estabilidad de las redes tróficas e interacciones ecológicas. Además, son un grupo diverso, con cerca de 1800 especies en el mundo ("World Register of Marine Species", WoRMS 2010), que incluyen a los anomuros pertenecientes a la superfamilia Paguroidea (Latreille 1802), los cuales presentan el abdomen total o parcialmente descalcificado y hacen uso de diversos resguardos para protegerlo. 
Los cangrejos ermitaños desarrollan diversas interacciones simbióticas con otras especies, al poner en recirculación los nutrientes que caen al fondo marino en forma de detritus y cuando generan sustratos estables mediante el uso de materiales calcáreos como refugio, tales como conchas vacías de moluscos o tubos de poliquetos. Por lo anterior, se les ha considerado como "ingenieros de los ecosistemas", debido a que favorecen el asentamiento de diversos epibiones, y desarrollan interacciones ecológicas complejas y únicas sobre la superficie de los refugios (Williams \& McDermott 2004).

Los aportes al conocimiento de este grupo en Colombia inlcuyen los siguientes trabajos: Campos (1978), quien registra 13 especies de cangrejos ermitaños, recolectados en la Bahía de Cartagena, y relaciona su presencia con algunas condiciones del sustrato y otros atributos ecológicos e indica además, que las familias más representativas son: Paguridae y Diogénidae. Sánchez \& Campos (1978), registran 16 especies pertenecientes a ocho géneros de las familias Paguridae y Coenobitidae. Lemaitre \& Campos (1993) describen las especies nuevas Pylopagurus pattiae y Xylopagurus tayrona, y establecen su presencia en otras regiones del Caribe. Campos \& Sánchez (1995), revisan los cangrejos ermitaños del género Paguristes y describen dos nuevas especies para la ciencia $P$. werdingi y P. zebra, además, aportan ocho registros nuevos para el Caribe colombiano.

A cargo del INVEMAR se realiza la caracterización de la fauna marina epibentónica en el talud superior del Caribe colombiano, con los cruceros Macrofauna I y II, de cuyas recolectas, se han derivado diversos estudios, orientados a profundizar el conocimiento sobre los crustáceos. Bermúdez (2000), Cruz (2001) y Campos et al. (2005), llevan a cabo la identificación taxonómica, cuantificación, distribución y estructura de la comunidad de los crustáceos decápodos obtenidos del crucero Macrofauna I, en donde se registran 16 especies de ermitaños recolectados entre $300-500 \mathrm{~m}$. Merchán et al. (2009) realiza un estudio sobre la composición de cangrejos ermitaños, recolectados por el crucero INVEMAR - Macrofauna II, en el cual se registró 177 individuos, pertenecientes a diferentes familias, 13 Paguridae, seis Diogenidae, dos Parapaguridae y un Pylochelidae y se describen patrones de distribución batimétricos, como agregaciones en los 20-70m, $70-150 \mathrm{~m}, 300-500 \mathrm{~m}$ y la influencia del río Magdalena como barrera ecológica.

Esta investigación determina los patrones de distribución de los cangrejos ermitaños con base en datos históricos, además de establecerse la composición de este grupo en el mar Caribe colombiano, mediante la clasificación y ordenación espacial de las comunidades, con una descripción de las especies características de los grupos, y la relación de estos patrones de distribución con zonas geográficas, ecológicas y paisajísticas ya existentes, las mismas permiten desarrollar un modelo para explicar las agrupaciones.

\section{MATERIALES Y MÉTODOS}

Área de estudio: El Caribe colombiano limita al este con Venezuela, en el sector de Castilletes y al oeste con Panamá, en el sector de Cabo Tiburón $71^{\circ} 18^{\prime}$ - $11^{\circ} 50$ ' N y $77^{\circ} 19^{\prime}$ $08^{\circ} 42^{\prime} \mathrm{W}$, tiene una longitud de línea de costa de 1 642km (Steer et al. 1997, Posada \& Rozo 2002) y su conformación actual es producto de diversos procesos orogénicos y volcánicos ocurridos por la actividad tectónica de las placas Suramericana y Caribe (Mejía 1989). Está influenciado por lo siguiente: (1) por la corriente del Caribe, que fluye en dirección noreste a lo largo del norte del Caribe y (2) por la circulación ciclónica del giro Panamá-Colombia (GPC) al suroeste; la parte del giro que linda con la costa se conoce como la contracorriente del Darién, la cual, genera un contraflujo continental desde Panamá hasta Venezuela. La corriente del Caribe es responsable de la surgencia de la Guajira durante la época seca y genera un hundimiento de la corriente del Darién en el suroeste del Caribe cuando los Alisios son máximos; esta convergencia de corrientes marinas y de vientos, junto con la posición de la costa, genera afloramientos de agua subsuperficial por transporte Ekman en 
el noreste; lo cual origina la formación de una piscina fría al noreste y una piscina cálida al suroeste (Andrade et al. 1988, Álvarez-León et al. 2003, Andrade et al. 2003).

Obtención y tratamiento de los datos: Los registros de las especies que incluyen coordenadas y localidades de recolecta, fueron obtenidas mediante bases de datos y referencias bibliográficas, incluyendo desde la primera publicación realizada por Pearce (1916) hasta los cruceros Macrofauna I, II y MacrofaunaCorpoguajira, este último realizado en el año 2006. Los ejemplares recolectados en los cruceros se encuentran depositados en el Museo de Historia Natural Marina de Colombia del INVEMAR, y su base de datos se puede acceder mediante la página www.Invemar.org.co. Estos registros fueron graficados y agrupados en 31 cuadrantes de medio grado por medio grado, mediante el programa ArcGIS ${ }^{\circledR} 9.2$ (Fig.
1) y utilizados para realizar una matriz de datos con atributos binarios de presencia (1) - ausencia (0). Los datos fueron analizados mediante métodos estadísticos multivariados, en donde se usó el software PRIMER-E V6 (Clarke \& Gorley 2006).

Para clasificar y ordenar espacialmente la comunidad, se calculó el índice de similitud de Jaccard, en el que se obtuvo un dendrograma probado mediante un test de perfiles de similitud SIMPROF, el cual, determinó cuáles ramificaciones fueron significativas y cuáles no. Para establecer las afinidades de los grupos y la continuidad o discontinuidad de los ensamblajes, fue aplicado un escalamiento no métrico multidimensional NMDS.

La existencia de diferencias entre las agrupaciones, se determinó planteando 10 hipótesis, de cómo podrían distribuirse las especies con base en mapas temáticos del mar Caribe colombiano: provincias geográficas, geomorfología

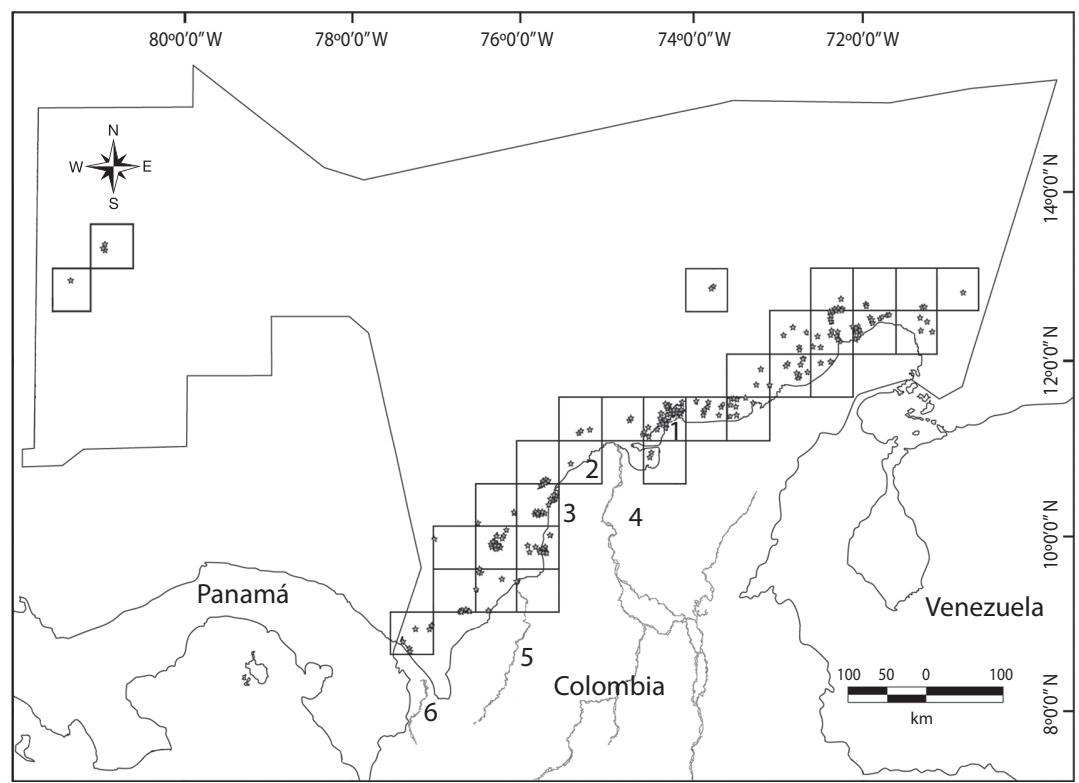

Fig. 1. Distribución de las especies de cangrejos ermitaños en el Mar Caribe colombiano, el área está dividida en 31 cuadrantes de medio grado por medio grado de dimensión. Convenciones: 1. Santa Marta; 2. Barranquilla; 3. Cartagena; 4. Río Magdalena; 5. Río Sinú; 6. Río Atrato; las estaciones están marcadas con una estrella.

Fig. 1. Distribution of the hermit crab species in the Colombian Caribbean Sea, the area was divided in 30 quadrants with half degree to half degree of dimension. Conventions: 1. Santa Marta; 2. Barranquilla; 3. Cartagena; 4. Río Magdalena; 5. Río Sinú; 6. Río Atrato; the stations are marked by a star. 
de los fondos marinos, profundidad, paisajes, ecozonas y ecorregiones (IDEAM et al. 2007); y características paleogeográficas, hidrodinámicas y climáticas basadas en los trabajos de Duque-Caro (1980), Blanco (1988) y ÁlvarezLeón et al. (2003). Para determinar el nivel de significancia de las agrupaciones, fue llevado a cabo un análisis de similitud ANOSIM, el mismo permitió evaluar las hipótesis sobre los patrones de distribución de los ermitaños.

Un análisis inverso de Kaandorp (1986), con las frecuencias relativas modificadas, permitió determinar las especies características de cada una de las agrupaciones obtenidas en el dendrograma de similitud. También, se usaron cuatro categorías según las frecuencias relativas (Fr), exclusivas: $100 \%_{(\mathrm{Fr})}$; muy representativas: $\leq 99 \%$ y $\geq 80 \%{ }_{(\mathrm{Fr})}$; representativas: $<80 \%$ $\mathrm{y} \geq 70 \%_{(\mathrm{Fr})}$ y generalistas: $<70 \%_{(\mathrm{Fr})}$.

Factores ambientales: Los factores ambientales fueron obtenidos mediante mapas temáticos de la serie: "ecosistemas continentales marinos y costeros de Colombia". Para crear los mapas, se usó una serie de variables físicas, oceanográficas y geomorfológicas, a partir de las cuales, se delimitaron como polígonos las zonas geográficas y ecológicas del Mar Caribe colombiano en una serie de mapas con distinto nivel de detalle: provincias geográficas, geoformas de los fondos marinos, paisajes, ecozonas y ecorregiones del mar Caribe colombiano (ver IDEAM et al. 2007).

Para el análisis, cada uno de estos mapas fue introducido como una capa y se asoció con las coordenadas espaciales de los registros de las especies mediante el programa ArcGIS ${ }^{\circledR} 9.2$ y se creó una matriz de datos, en donde se relacionaba cada polígono con los registros de las especies que se encontraban sobre el mismo, y se obtuvo un total de 46 factores (Cuadro 1). Asimismo, se estableció la relación de los factores ambientales con la distribución de las especies, se aplicó el procedimiento BVSTEP (Clarke \& Ainsworth 1993, Clarke \& Gorley 2006). Después de que se obtuvieron los factores, se buscó en el trabajo "ecosistemas continentales marinos y costeros de
Colombia" qué aspectos fueron utilizados para crear dicho polígono, y de esta forma saber qué variables son las que están determinando las agrupaciones.

Relación de los patrones de distribución locales respecto a los globales: Con el fin de determinar qué regiones del Caribe y el Atlántico son más afines con la fauna colombiana, se identificó la distribución mundial de las especies registradas en Colombia, y se realizó una matriz de datos de presencia-ausencia en las subprovincias faunísticas propuestas para el gran Caribe y el Atlántico noreste por SalazarVallejo (2000), además, se incluyó un grupo llamado Atlántico sur que comprende desde Uruguay hasta Argentina. Con base en la matriz de datos se sumó el total de especies en común con Colombia por subprovincia y se obtuvo un porcentaje de acuerdo con el total de especies registradas en Colombia. Posteriormente, se determinó cuánto de ese porcentaje, se distribuía ampliamente por todo el Caribe y cuánto tenía una distribución restringida.

\section{RESULTADOS}

La matriz usada para realizar el análisis de clasificación (Jaccard), solo incluyó 30 de los 31 cuadrantes originales, puesto que se eliminó el número 25 , debido a que en él se registraba una sola especie sin más registros, por lo cual el cuadrante era poco informativo. Ahora bien, se identificaron ocho grupos (SIMPROF; Fig. 2a), representados con una letra de la $\mathrm{A}$ a la $\mathrm{H}$ : el A presenta un 5\% de similitud y el B un $20 \%$ (Fig. 2b), y juntos forman el sector noreste (NE) del Caribe colombiano, que incluye las ecorregiones Guajira y Palomino; el grupo C con una similitud del $42 \%$, corresponde a Santa Marta, Cartagena, Islas del Rosario e Islas de San Bernardo, ubicadas en el centro (CEN) del Caribe colombiano; el grupo D tiene de similitud un $55 \%$, pertenece al sector suroeste (SO) que incluye la ecorregión Darién y parte de la ecorregión Morrosquillo; el grupo E con un $67 \%$ de similitud, corresponde al sector de San Andrés y Providencia; en el grupo F se 


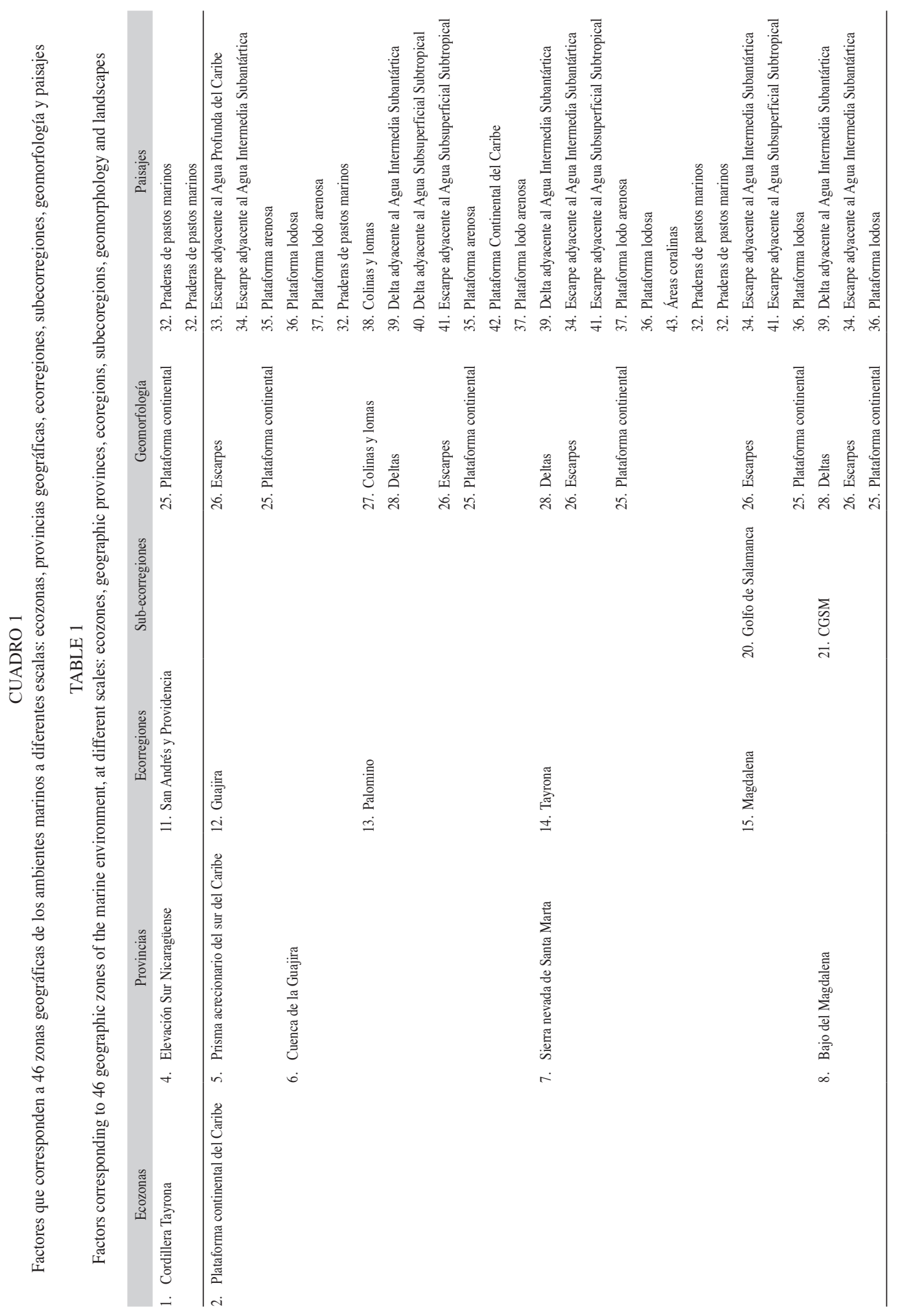




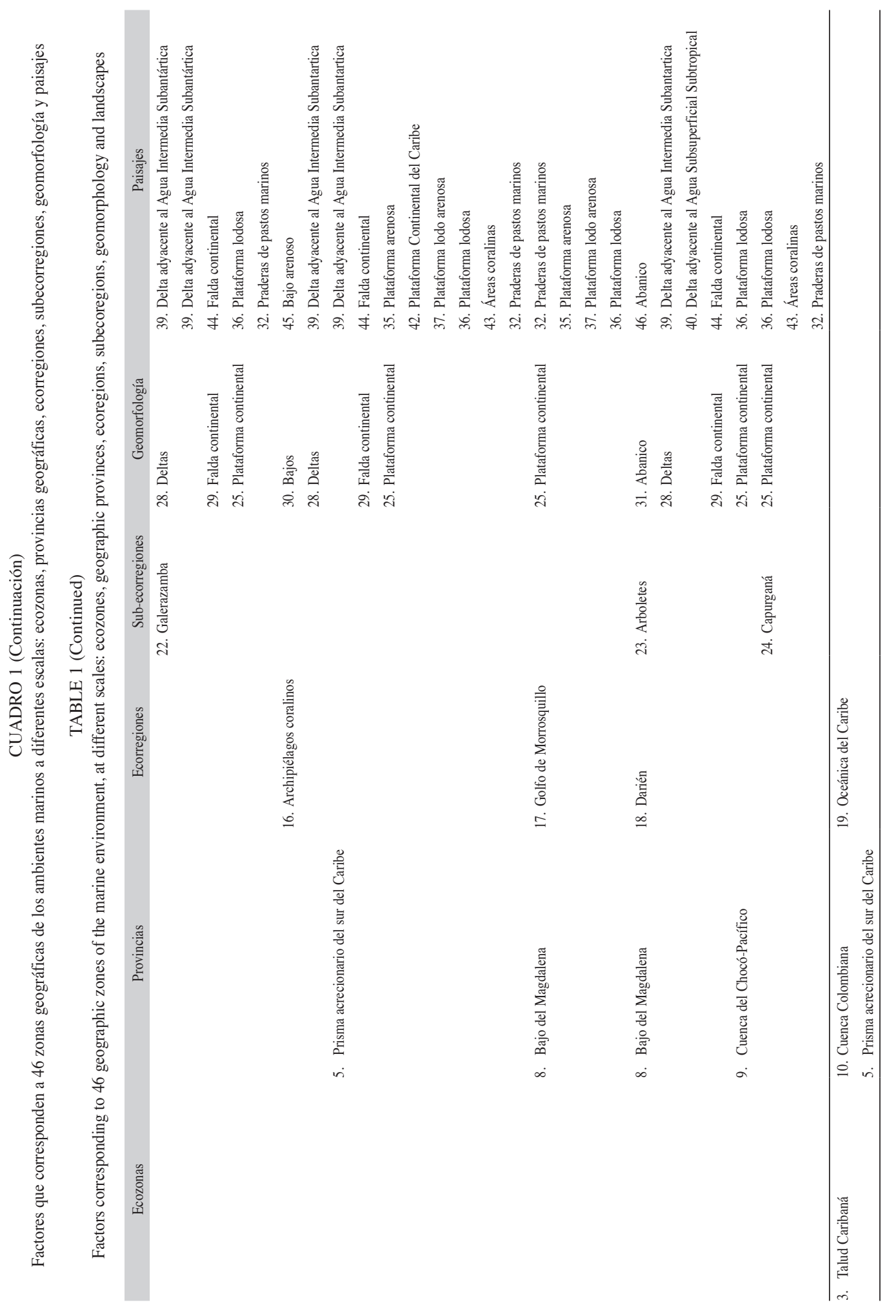


A

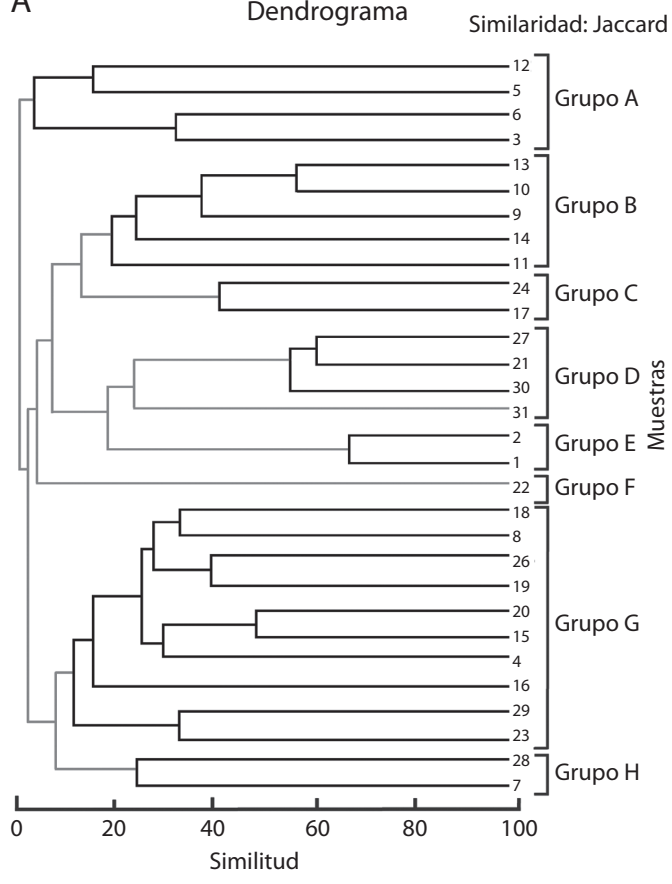

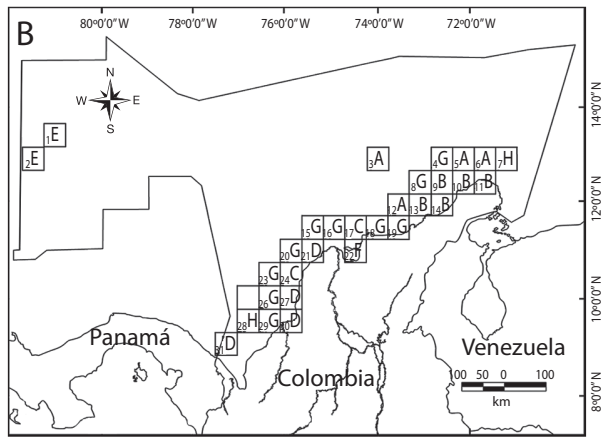

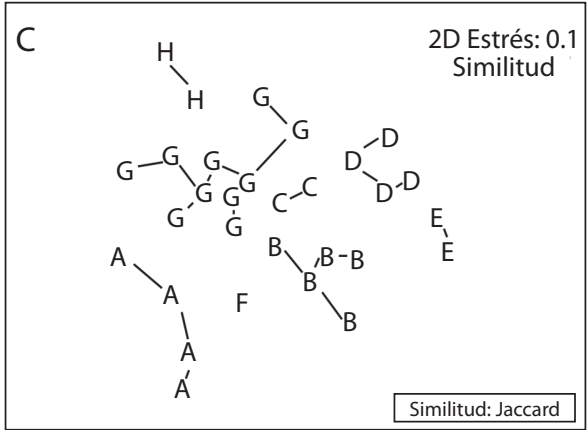

Fig. 2. (A) Dendrograma con una agrupación presencia/ausencia de las especies sobre los cuadrantes, que forma ocho grupos (A-H), el eje X representa el porcentaje de similtud, las líneas negras muestran las agrupaciones que son significativas según el análisis SIMPROF (B) Distribución geográfica de los grupos, las letras coinciden con las agrupaciones del dendrograma y el NMDS (C) Disposición espacial de las estaciones (NMDS), las letras muestran los grupos formados en el dendrograma. Fig. 2. (A) Cluster by presence/absence of the species associated to the quadrants, forming eight groups (A to H), the $X$ axis represent the percents the percentage of similarity, the black lines show the different groups formed by the SIMPROF analyses (B) Geographic distribution of groups, the drafts matching with the groups in the resemblance and the NMDS. (C) NMDS showing the spatial arrangement of the stations, the drafts represent the groups formed in the dendrogram.

encuentra la Ciénaga Grande de Santa Marta. El grupo G con una similitud del $12 \%$ abarca las estaciones que se encuentran sobre o cerca al talud continental, e indica que en aguas más profundas, las especies conforman un ensamblaje particular que difiere de aquellos formados en aguas someras; los cuadrantes del grupo $\mathrm{H}$ parecen no presentar continuidad espacial, aunque se conforman por cuadrantes dispuestos en aguas oceánicas.

En el NMDS, se observa una distribución estrecha, debido a que los grupos se encuentran próximos entre sí, pero bien diferenciados, (Fig. 2c), en donde se muestra al sector noreste hacia la parte inferior izquierda (Grupos A, B y F), el suroeste hacia la derecha (Grupos D y E), el que está compuesto por las estaciones cercanas o sobre el talud continental en la parte superior (Grupos G, H) y un cuarto sector representado por las zonas que van de Cartagena al Tayrona (Grupo C) justo en el centro de la gráfica, y genera un gradiente de distribución con los otros grupos.

De los ocho grupos formados en el dendrograma, A, B, C, D y G presentan especies particulares (Kaandorp $\geq 70 \%_{(\mathrm{Fr})}$ ), (Cuadro 2), las que se encuentran en los grupos E, F y $\mathrm{H}$, presentaron frecuencias relativas $\leq 50 \%$. El total de especies generalistas fue de 33, de las cuales, Dardanus fucosus, Clibanarius 
CUADRO 2

Especies más importantes dentro de los grupos formados en el dendrograma de similitud según los resultados obtenidos del análisis inverso de Kaandorp

TABLE 2

Most important species within the groups formed in the dendrogram of similarity according to the results obtained from the inverse analyses of Kaandorp

\begin{tabular}{|c|c|c|c|c|}
\hline Grupo A & Grupo B & Grupo C & Grupo D & Grupo G \\
\hline \multicolumn{5}{|c|}{ Exclusivas $100 \%(\mathrm{Fr})$} \\
\hline Paguristes inconstans & $\begin{array}{l}\text { Manucomplanus ungulatus } \\
\text { Pagurus leptonyx }\end{array}$ & $\begin{array}{l}\text { Isocheles wurdermanii } \\
\text { Paguristes cadenati } \\
\text { Paguristes grayi } \\
\text { Paguristes starcki } \\
\text { Paguristes tortugae } \\
\text { Paguristes werdingi } \\
\text { Paguristes zebra } \\
\text { Pagurus carolinensis } \\
\text { Pagurus marshi } \\
\text { Pylopagurospis atlantica }\end{array}$ & $\begin{array}{l}\text { Calcinus urabaensis } \\
\text { Paguristes lapillatus }\end{array}$ & $\begin{array}{l}\text { Paguristes limonensis } \\
\text { Paguristes sp. } 1 \\
\text { Parapagurus alaminos } \\
\text { Parapagurus } \\
\text { pilosimanus } \\
\text { Pylopagurus macgeorgei } \\
\text { Tomopaguropsis sp }\end{array}$ \\
\hline \multicolumn{5}{|c|}{ Muy representativas: $\leq 99 \%$ y $\geq 80 \%$ (fr) } \\
\hline & & & & $\begin{array}{l}\text { Oncopagurus gracilis } \\
\text { Tomopagurus chacei } \\
\text { Xylopagurus tayrona }\end{array}$ \\
\hline \multicolumn{5}{|c|}{ Representativas: $<80 \%$ y $\geq 70 \%$ (fr) } \\
\hline & $\begin{array}{l}\text { Iridopagurus margaritensis } \\
\text { Paguristes hummi }\end{array}$ & & & $\begin{array}{l}\text { Paguristes sp. } 2 \\
\text { Pylopagurus discoidalis }\end{array}$ \\
\hline
\end{tabular}

antillensis, Pagurus brevidactylus, Paragiopagurus pilimanus, Phimochirus operculatus y Petrochirus diogenes fueron las que se encontraron más ampliamente distribuidas en el mar Caribe colombiano, y estan presentes en cuatro o cinco de los grupos.

El modelo con la mejor representación de las agrupaciones es aquel que combina la distribución noreste, centro, suroeste, basada en la división propuesta por Duque-Caro(1980), con una segregación relacionada con la profundidad (Cuadro 3, Fig. 3) ANOSIM, R global de 0.527 , el cual, indica que las agrupaciones se forman a pesar que los grupos tienden a superponerse (Fig. 4).

Composición de acuerdo a la profundidad: En el talud (grupo G, Fig. 2) se presentaron seis especies exclusivas, de la cuales tres son reconocidas a nivel mundial, por distribuirse en aguas profundas, estas son: Parapagurus pilosimanus, registrada entre 102 y $3864 \mathrm{~m}$, es más frecuente entre 400-1 400m (Lemaitre 1989), Parapagurus alaminos que se distribuye entre 850-3 360m (Lemaitre 1989) y Pylopagurus macgeorgei que se distribuye entre 453-715m (McLaughlin \& Lemaitre 2001). Paguristes sp. 1 y Tomopaguropsis sp. (ver descripción en Martínez 2010), presentes entre $312-500 \mathrm{~m}$ y $296-464 \mathrm{~m}$, respectivamente, son endémicas, de las aguas del talud del Mar Caribe colombiano. Paguristes limonensis es registrada en el Caribe desde los 18-234m, por lo que si bien en Colombia solamente se encuentra en aguas profundas, no es una especie exclusiva de estas zonas.

Composición de acuerdo a los sectores: Con base en los resultados obtenidos en el ANOSIM y en los grupos que presentaron 


\section{CUADRO 3}

Resultados del ANOSIM para las diferentes hipótesis propuestas para explicar las agrupaciones obtenidas mediante el dendrograma de similitud

TABLE 3

ANOSIM results for the different hypotheses proposed to explain the cluster obtained by the dendrogram of similarity

\begin{tabular}{|c|c|c|c|c|}
\hline $\mathrm{H}_{0}$ & $\mathrm{H}_{\mathrm{a}}$ & $\mathrm{P}$ & R global & Resultados \\
\hline \multirow{10}{*}{$\begin{array}{l}\text { Las } \\
\text { agrupaciones } \\
\text { no se } \\
\text { diferencian } \\
\text { lo suficiente } \\
\text { como para } \\
\text { considerar } \\
\text { que existe } \\
\text { un patrón de } \\
\text { distribución }\end{array}$} & Las agrupaciones coinciden con la geomorfología del mar Caribe Colombiano & 0.265 & 0.066 & Acepto $\mathrm{H}_{0}$ \\
\hline & Las agrupaciones coinciden con los paisajes del mar Caribe colombiano & 0.123 & 0.207 & Acepto $\mathrm{H}_{0}$ \\
\hline & Las agrupaciones coinciden con las provincias geográficas del Caribe & 0.199 & 0.077 & Acepto $\mathrm{H}_{0}$ \\
\hline & Las agrupaciones coinciden con las ecorregiones & 0.581 & 0.027 & Acepto $\mathrm{H}_{0}$ \\
\hline & Las agrul & 0.003 & 0.235 & Acepto $\mathrm{H}_{0}$ \\
\hline & Las agrupaciones coinciden con patrón de distribución noreste-suroeste & 0.001 & 37 & Acepto $\mathrm{H}_{0}$ \\
\hline & Las agrupaciones coinciden con patrón de distribución noreste-centro-suroeste & 0.001 & 0.188 & Acepto $\mathrm{H}_{0}$ \\
\hline & Las agrupaciones coinciden con patrón de distribución por profundidad & 0.001 & 0.311 & Rechazo $\mathrm{H}_{0}$ \\
\hline & Las agrupaciones coinciden con patrón de distrib. noreste-suroeste/profundidad & 0.001 & 0.504 & Rechazo $\mathrm{H}_{0}$ \\
\hline & Las agrupaciones coinciden con patrón de distrib. noreste-centro-suroeste/profundidad & 0.001 & 0.527 & Rechazo $\mathrm{H}_{0}$ \\
\hline
\end{tabular}

Valores por debajo de 0.25 son considerados como poco significativos.

Values underlie 0.25 was considerate insignificant.

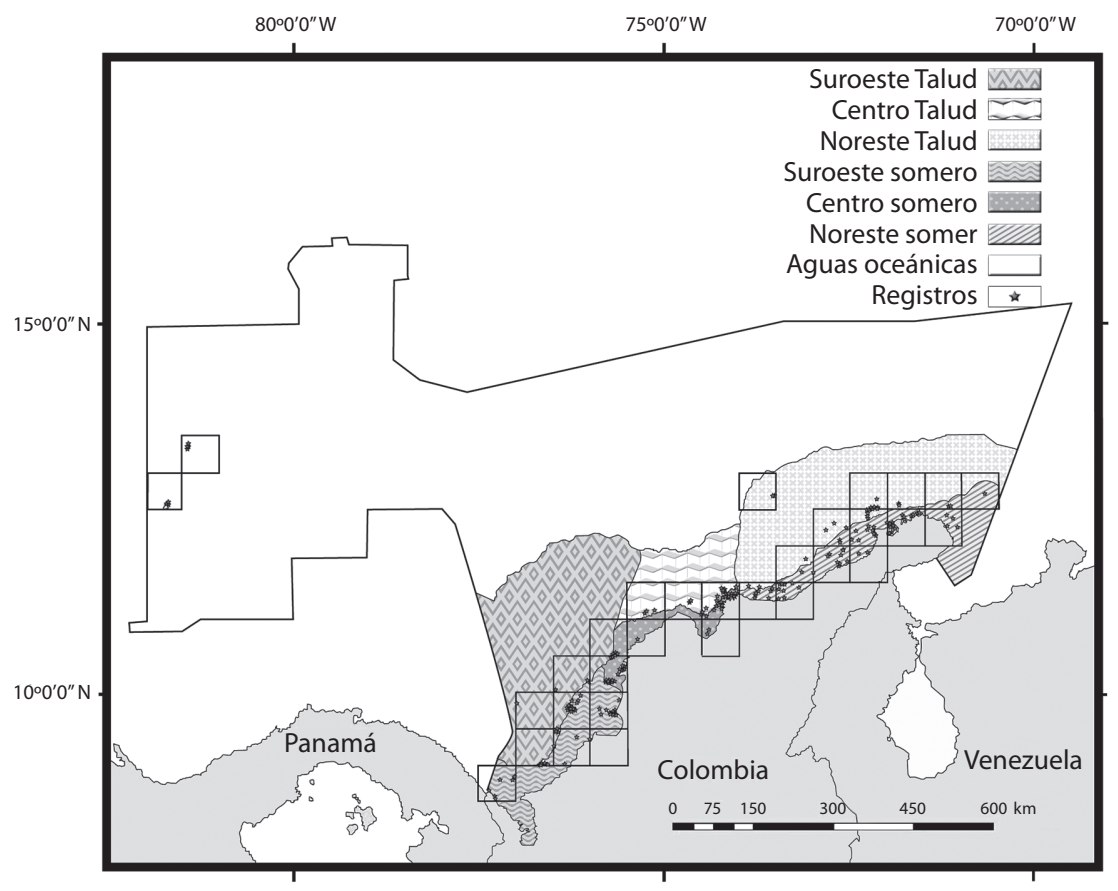

Fig. 3. Con base en la unión de los mapas de Duque-Caro (1980) y la relación de la profundidad con los patrones de distribución, se crea el mapa que mejor representa el patrón de distribución de los cangrejos ermitaños en al mar Caribe colombiano.

Fig 3. Based in the union of the maps from Duque-Caro (1980) and the relationship between the Depth and the patterns of distribution was made a map that represent the better pattern of distribution of the hermit crabs in the Colombian Caribbean Sea. 


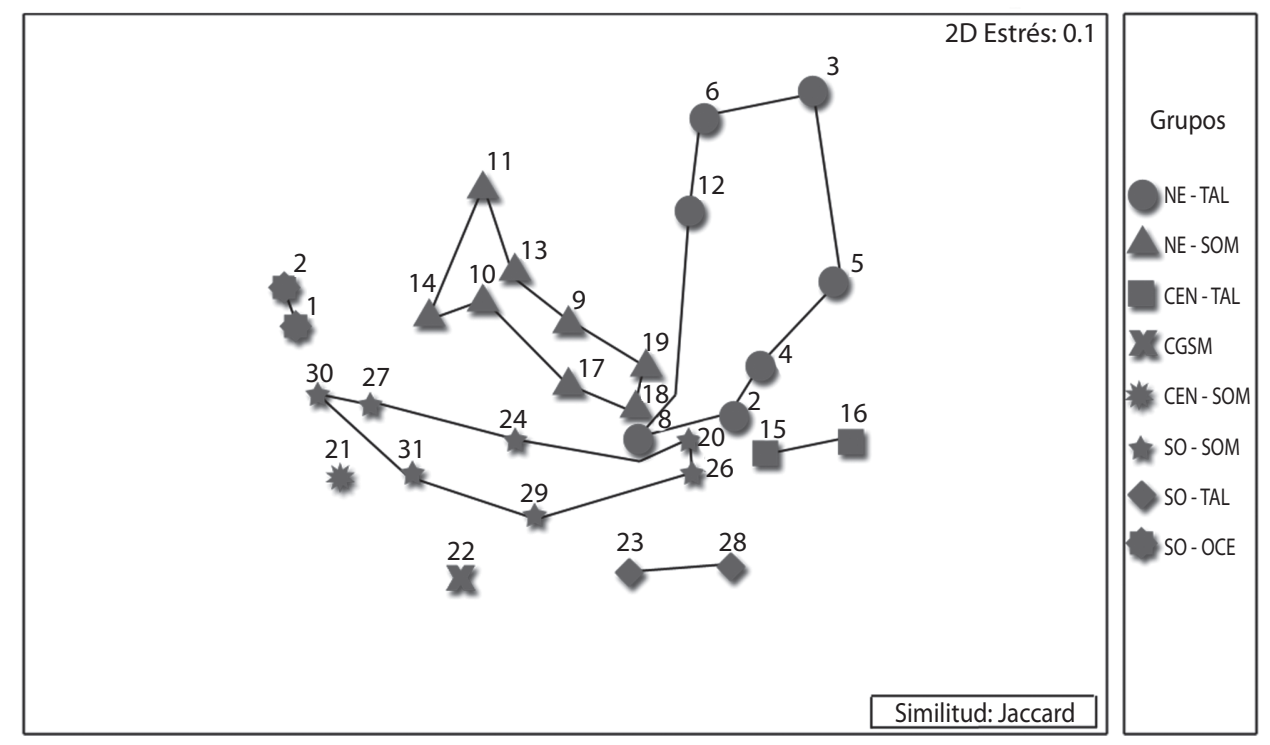

Fig. 4. NMDS, agrupaciones obtenidas por el análisis de similitud respecto al patrón de distribución, nótese que aunque los grupos se observan claramente, existe una superposición del sector noreste cercano al talud continental con el sector noreste somero y suroeste somero. Acotaciones: NE (noreste); CEN (centro); SO (suroeste); SOM (somero); TAL (cercano al talud continental); OC (aguas oceánicas) y CGSM (Ciénaga Grande de Santa Marta).

Fig. 4. NMDS, showing the cluster obtained by the similarity analysis regarding the distribution pattern, note that although groups are clearly observed, there is overlap near to the Northeast sector nearly to the continental slope and to the Northeast and Southwest shallow. Notes: NE (Northeast); CEN (center); SO (Southwest); SOM (Shallow); TAL (near to the continental slope); OC (oceanic waters) y CGSM (Grande of Santa Marta Swamp).

especies exclusivas en el análisis de Kaandorp, se destacaron tres grupos costeros:

Noreste: (grupos A y B) presentaron cuatro especies exclusivas, una en el grupo A, dos en el grupo B y una en el grupo A y B. Paguristes inconstans se encuentra en el talud de la Guajira (grupo A) a $337 \mathrm{~m}$ de profundidad, se encuentra en el área continental desde la Florida hasta Colombia y en las Antillas Menores, pero no se registra en las Antillas Mayores (McLaughlin \& Provenzano 1974b). Las especies del grupo B, se hallaron en la plataforma continental de la Guajira, son nuevos registros para Colombia: Manucomplanus ungulatus con una distribución amplia dentro del Atlántico tropical occidental, Carolina del Norte, Florida, las Bahamas y el golfo de México (Lemaitre \& McLaughlin 1996) y Pagurus leptonyx el cual solamente se registra en las costas de Brasil a profundidades entre 10-20m (Lemaitre et al. 1982); Iridopagurus margaritensis (grupos A y B), se distribuye ampliamente sobre el Caribe, desde las Bahamas hasta la Guyana Francesa, e incluyen las Antillas Mayores y Menores (García-Gómez 1983). De igual manera, se presentaron tres especies representativas: Paguristes hummi recolectada entre el sector NE y CEN del Mar Caribe colombiano, a nivel mundial se distribuuye en la Florida (Abele \& Kim 1986) y Paguristes paraguanensis que se encuentra entre el NE y CEN del Caribe colombiano, Venezuela y Suriname (McLaughlin \& Provenzano 1974a).

Centro: En el sector centro del Caribe colombiano (grupo C) están presentes diez especies exclusivas, algunas con un alto grado de endemismo, Paguristes werdingi y P. zebra solamente han sido registradas en Colombia 
por Campos \& Sánchez (1995); Pagurus carolinensis se distribuye desde el Atlántico norte hasta Colombia (McLaughlin 1975, Lemaitre et al. 1982); Paguristes cadenati y Pagurus marshi desde el Atlántico norte hasta Curaçao (Lemaitre et al. 1982); mientras que Pylopaguropsis atlantica, únicamente ha sido registrada fuera de las costas de Florida, en Colombia y Surinam (Wass 1963, McLaughlin \& Haig 1989, Campos et al. 2005). En todos los casos, la subprovincia Colombo-Venezolana, propuesta por Díaz (1995) y modificada por Salazar-Vallejo (2000), es el límite geográfico austral de estas especies, lo cual indica cierto grado de endemismo. Isocheles wurdermanni se distribuye ampliamente desde el Gran Caribe hasta Brasil, mientras que Paguristes tortugae y P. starcki en el Caribe y el Atlántico (Provenzano 1965, McLaughlin \& Provenzano 1974b).

Suroeste: (Grupo D) solamente se encontraron dos especies exclusivas, con un alto grado de endemismo: Calcinus urabaensis, endémica del suroeste del Caribe colombiano y Paguristes lapillatus, que solo ha sido registrada en Colombia y Honduras (McLaughlin \& Provenzano 1974b).

Factores ambientales: Asimismo, fueron ocho los factores determinantes en los patrones de distribución (Fig. 5). El Talud Caribaná, es la ecozona que representa el efecto de la profundidad en los ensamblajes; en la cual, variables como la temperatura, contenido de oxígeno y salinidad, pueden cambiar por la

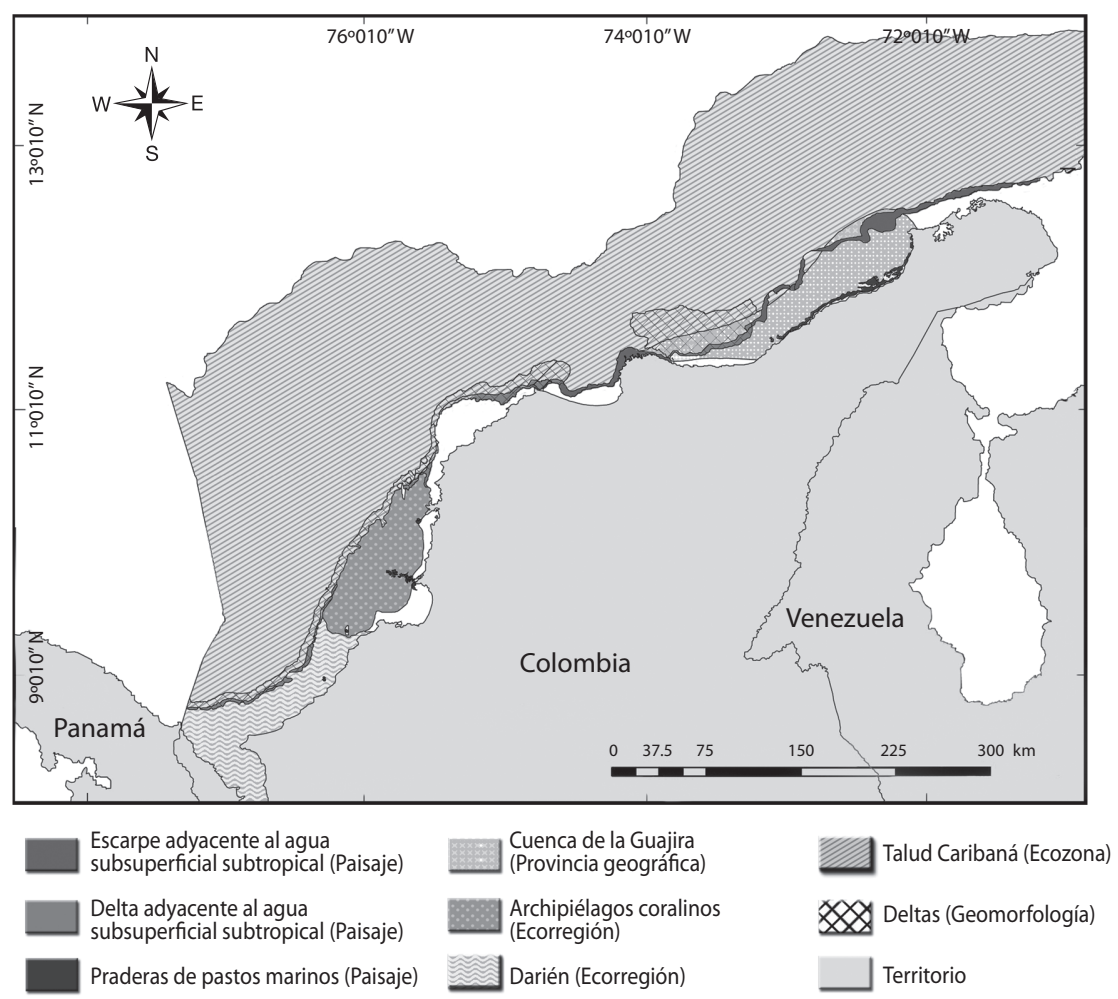

Fig. 5. Selección de los factores que mejor explican los patrones de distribución de los cangrejos ermitaños en el mar Caribe colombiano, estos incluyen un aspecto geomorfológico, tres paisajes, dos ecorregiones, una provincia geográfica y una ecozona.

Fig. 5. Selected factors that best explain the distribution patterns of hermit crabs in the Colombian Caribbean Sea, these include a geomorphologic aspect, three landscapes, two ecorregiones, a geographic province and ecozone. 
distancia a la costa y por eventos oceanográficos estacionales como las surgencias (Wenner \& Boesch 1979); las manifestaciones de estos eventos, son las masas de agua, las cuales, son entidades físicas de volumen conocido, con una historia común en su formación y que ocupan un volumen finito (Tomczak 1999); los paisajes son producto de la relación entre las masas de agua y los rasgos geomorfológicos que yacen bajo éstos, y dos de estos representan la termoclina del Mar Caribe colombiano: el "Escarpe adyacente al agua subsuperficial subtropical y delta adyacente al agua subsuperficial subtropical", que forman una corriente bajo el centro de surgencias cuyo núcleo de máxima velocidad se desplaza a $200 \mathrm{~m}$ de profundidad, con temperaturas superficiales entre $22.6^{\circ} \mathrm{C}-25.8^{\circ} \mathrm{C}$, que pueden descender hasta $19^{\circ} \mathrm{C}$ en los focos de surgencia activa, y salinidades altas entre 36.537.2\%o (Críales-Hernández et al. 2003, Bernal et al. 2006, IDEAM et al. 2007).

Las praderas de pastos marinos son asociaciones de plantas vasculares, entre las que se encuentra Thalassia testudinum, Syringodium filiforme, Halodule wrightii y el género Halophila; se relacionan con una alta productividad atribuida a la unión entre el metabolismo propio de las praderas con la producción primaria de las algas asociadas y son reconocidas por ser de gran importancia para los crustáceos (IDEAM et al. 2007). Se encuentran en 17 zonas geográficas del Caribe colombiano con una distribución discontinua, destacándose las que yacen sobre la Cuenca de la Guajira, que representan más del $80 \%$ del total del Caribe colombiano (Santodomingo et al. 2004), la praderas de esta región son consideradas como ecosistemas inmaduros por las grandes tasas de producción primaria que son poco aprovechadas en los niveles tróficos superiores (CríalesHernández et al. 2003).

En la Ecorregión Archipiélagos Coralinos, el elemento geomorfológico más relevante es la presencia de domos diapíricos colonizados por arrecifes, el cual conforma los archipiélagos del Rosario y San Bernardo (IDEAM et al. 2007). Las áreas coralinas por lo general, presentan biotopos y hábitats asociados, distribuidos en mosaico, distinguidos entre sí por la naturaleza física del sustrato (sedimentos, escombros coralinos, rocas) y por los componentes bióticos que cubren el fondo (algas, fanerógamas, esponjas, abanicos de mar, entre otros) (Díaz \& Gómez-López 2000). Hacia el suroeste, en la Ecorregión Darién las praderas de pastos marinos son más escasas que al noreste, los sustratos son lodosos e influenciados por la descargas de los ríos Atrato y Sinú, y no se presentan eventos costeros de surgencia, por lo que, las aguas son más cálidas (Díaz \& Gómez-López 2000), y muestran un ecosistema completamente distinto que alberga especies estenotípicas de cangrejos ermitaños.

Relación de los patrones de distribución locales respecto a los globales: Las subprovincias Colombo-Venezolana y Nica-Colombiana (Fig. 6) son las más afines con la fauna de ermitaños registrada en Colombia, y se relaciona en un 21 y $16 \%$ respectivamente; a éstas les siguen la provincia Floridiana del Caribe norte y Carolinense del Atlántico norte con un

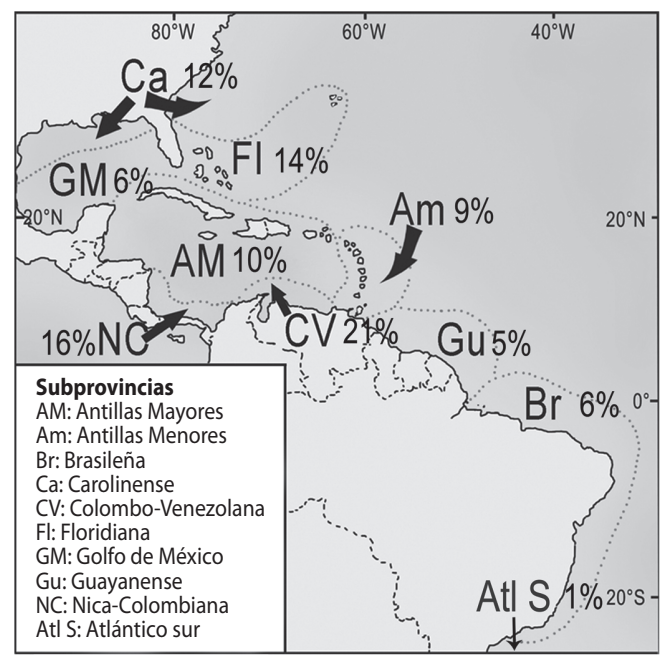

Fig. 6. Afinidades de las provincias y subprovincias del Atlántico oeste (Modificado de Salazar-Vallejo 2000) con la fauna de cangrejos ermitaños registrada para el mar Caribe colombiano.

Fig. 6. Affinities of the provinces and subprovinces of the western Atlantic (Modified Salazar-Vallejo 2000) with the hermit crabs from Colombian Caribbean Sea. 
14 y $12 \%$, respectivamente; la región insular del Mar Caribe comprendida por las Antillas mayores (10\%) y menores (9\%) les siguen en afinidad a las subprovincias del norte, las especies del Golfo de México tienen poca afinidad con las colombianas, y se comparte solamente un $6 \%$ del total registrado; las provincias al sur del Caribe, Guyanas y Brasil, presentaron poca afinidad (5 y 6\%, respectivamente), finalmente el Atlántico sur mostró muy poca afinidad $(1 \%)$, lo que indica que la fauna colombiana se relaciona mucho más con la fauna del Atlántico norte y la región septentrional del Mar Caribe que con el Atlántico austral.

Sin embargo, al observar el grado de distribución de las especies y su composición dentro de las subprovincias, se observa que el $58.2 \%$ de las especies presentes en Colombia, se encuentran ampliamente desde el Atlántico norte hasta el Atlántico sur (Fig. 7), el 26.2\% se estan presentes desde el Atlántico norte hasta la subprovincia Colombo-Venezolana, el 9.1\% de las especies se dan desde el Atlántico sur hasta la subprovincia Nica-Colombiana y el $6.5 \%$ de las especies son endémicas de las subprovincias presentes en el Mar Caribe colombiano: Nica-Colombiana, Antillas Mayores (que toca el Caribe colombiano en el sector de Santa Marta) y Colombo-Venezolana, por lo que es probable que estas provincias sean una zona de transición dentro del Atlántico norte y sur.

\section{DISCUSIÓN}

Composición de acuerdo con la profundidad: En el talud Caribaná ocurren cambios en la termoclina, los cuales están mediados por las masas de agua subsuperficiales subtropicales que son determinantes en la composición de las especies en las aguas profundas, las cuales, están relacionadas con cambios en la salinidad y contenido de oxígeno del agua

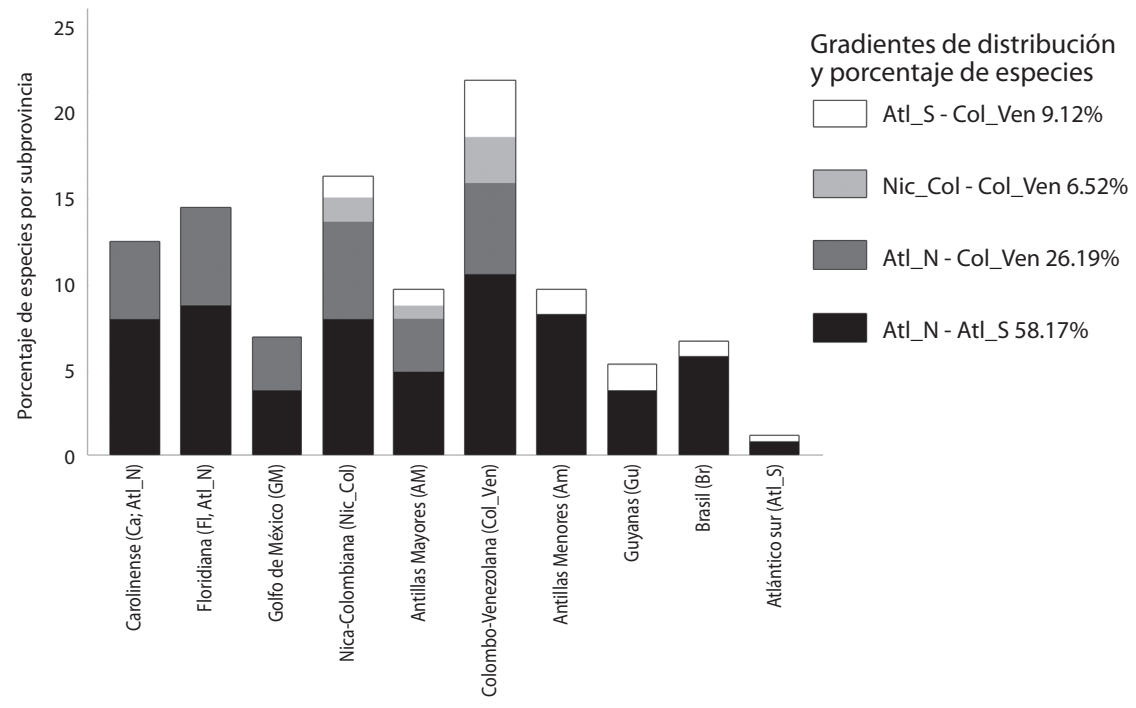

Subprovincias del Atlántico y el mar Caribe

Fig. 7. Histograma de frecuencias del gradiente de distribución de las especies en el mar Caribe mediante bandas (eje X); el cuadro de la derecha indica los límites de las subprovincias y el porcentaje de especies que se distribuyen entre esos límites; El eje Y corresponde al porcentaje de especies registradas en cada subprovincia.

Fig. 7. Frequencies histogram showing the gradient of distribution of the species in the Caribbean Sea through bands (X axis); the right square, pointed the boundaries of the subprovinces and the percent of the species that is distributing between this boundaries; the $\mathrm{Y}$ axis represent to the percent of species in each subprovinces. 
y son consideradas por varios autores como determinantes en los patrones de distribución por profundidad de los crustáceos (Pequegnat \& Pequegnat 1970, Wenner \& Boesh 1979, Hendrix 2003, Navas et al. 2003, Bermúdez 2007). Estos cambios a nivel local, ocurren porque conforme la profundidad aumenta, se disminuye la velocidad de las corrientes y decrece la influencia de los vientos Alisios (Bermúdez 2007). Por el contrario, en las zonas costeras, la influencia de los ríos Magdalena, Sinú, Atrato y otros tributarios menores, son los causantes principales de que las condiciones de temperatura, salinidad y oxígeno disuelto sean diferentes en aguas someras que en aguas profundas, y se convierte en un factor determinante en los patrones de distribución locales de los cangrejos ermitaños (Merchán et al. 2009).

Varias especies de otros grupos marinos también presentan este mismo patrón de distribución relacionado con la profundidad, entre ellas los peces (Wenner \& Boesh 1979); poliquetos y bivalvos (Sanders \& Hesler 1969); y gasterópodos (Rex 1977), estos últimos se encuentran relacionados estrechamente con los ermitaños, los cuales, usan las conchas vacías como fuente principal para obtener refugio. La disponibilidad de conchas en el medio, ha sido considerada como un factor limitante dentro de las poblaciones de los ermitaños y muchas especies que son muy selectivas solamente escogen conchas de una especie o forma determinada, lo cual, puede ejercer un efecto restrictivo en la distribución de algunas especies (Bertness 1981, Mantellato \& Dominiciano 2002, Williams \& McDermott 2004, Merchán et al. 2009). La preferencia de ciertos tipos de concha, además proveen al cangrejo las mismas ventajas que al gasterópodo, por ejemplo, $\mathrm{Cli}$ banarius antillensis que se encuentra en hábitats con una alta dinámica intermareal, prefiere conchas altamente espiraladas que maximizan la protección contra el estrés térmico; mientras que Calcinus tibicen evita la emersión por las mareas y prefiere conchas poco espiraladas y duras que mejoran su resistencia hacia predadores frecuentes en hábitats como los arrecifes de coral (Bertness 1981).
La relación con eventos oceanográficos y climáticos le da una cierta temporalidad a las barreras geográficas, que determinan los patrones de distribución de las especies asociadas al talud Caribaná, por lo cual, hacia aguas más profundas los cangrejos ermitaños presentan un patrón de distribución en gradiente, relacionado con un alto grado de conectividad, lo cual permite mayor potencial de dispersión de las larvas debido a la homogeneidad de las condiciones ambientales y los ecosistemas (Bermúdez 2000); no obstante, dicha conectividad puede verse temporalmente interrumpida por dos razones: (1) Existe un rezago de aproximadamente cinco meses entre los periodos climáticos del noreste y suroeste del mar Caribe colombiano, por lo tanto podrían existir ligeras asincronías entre los periodos de reproducción de las poblaciones (Bernal et al. 2006); (2) según Bermúdez et al. (2008) la conectividad entre las poblaciones puede facilitarse o inhibirse, y depende de la época y estrategias reproductivas de cada una de las especies, por lo que estrategias de reproducción estacionales relacionadas con la época seca, harían que las larvas fueran retenidas por los giros ciclónicos, mientras que estrategias de reproducción continua permitirían el flujo de las larvas, de esta forma propician una mayor distribución, y explican por qué el sector noreste del talud (grupo A) forma un grupo diferente al del talud del resto del Caribe colombiano (grupo G) (Fig. 3).

Composición de acuerdo a los sectores: La sectorización latitudinal en las aguas costeras, se hace más evidente debido a que los nichos son más variables a menor profundidad, de tal manera que los individuos se especialicen para poder sobrevivir en las condiciones particulares de los ecosistemas en los que habitan, y se restringe su potencial de dispersión (Gherardi 1990).

El sector noreste, se caracteriza por las grandes tasas de producción primaria debida a las praderas de pastos marinos de la Guajira; en estos ecosistemas, los detritívoros y excavadores, como los cangrejos ermitaños, desempeñan 
un papel importante, al poner los nutrientes que se precipitan en el sedimento en recirculación, y así favorecer la asociación de comunidades que hacen uso de estos recursos que por lo general son poco aprovechados, lo anterior contribuye con el mantenimiento de las redes tróficas de las praderas y nichos circundantes, que usan estos nutrientes (Criales-Hernández et al. 2003, Williams \& McDermott 2004).

En el sector centro está el mayor número de especies exclusivas, puesto que esta región presenta características particulares que la distinguen de los sectores del NE y SO. Una posible explicación a este hecho es: el sector es un mosaico que alberga una amplia diversidad de ecosistemas, entre ellos los estuarios, ciénagas y lagunas formadas por el efecto del río Magdalena; litorales rocosos formados por las estribaciones de la Sierra Nevada de Santa Marta y por las plataformas calcáreas en la región de Cartagena; arrecifes de coral y praderas de pastos marinos que se encuentran en el Parque Tayrona e Islas del Rosario; bosques de manglar de los sistemas deltaicos del río Magdalena, Parque Nacional Natural Tayrona (PNNT), Cartagena e Islas del Rosario; igualmente, en el PNNT existe la mayor diversidad de macroalgas del Mar Caribe colombiano, que son de gran importancia para el bentos; este mosaico de paisajes permite albergar una mayor diversidad de especies adaptadas a ecosistemas particulares (Díaz \& Acero 2003, Díaz-Pulido \& Díaz-Ruíz 2003).

Las principales características del sector suroeste son la turbidez de las aguas, los sustratos de tipo lodoso, los bajos niveles de salinidad y estrechez de la plataforma continental (Díaz 1995, Salazar-Vallejo 2000). Asimismo, se relaciona con un nivel de conectividad bajo debido al efecto del giro ciclónico de Panamá-Colombia que aumenta la tasa de auto reclutamiento de las larvas, lo cual restringe las poblaciones locales del resto del Caribe (Cowen et al. 2006, Bermúdez 2005, 2007).

En un plano bidimensional (NMDS), las agrupaciones muestran una distribución estrecha, en la que los grupos se encuentran próximos entre sí, pero bien diferenciados, lo mismo indica un efecto de gradiente; por lo cual, aunque existen patrones de distribución definidos, estos presentan límites difusos, que pueden atribuirse a un número abundante de especies euritípicas, que al habitar diferentes ecosistemas, presentan una distribución más amplia. Estas especies, aumentan el grado de correlación entre las agrupaciones que ocurren en áreas pequeñas como el Mar Caribe colombiano, donde las unidades biogeográficas corresponden a centros de endemismos, ecorregiones o subprovincias, en las cuales, los grupos tienden a presentar mayores afinidades con grupos cercanos espacialmente, y presentan menores porcentajes de especies endémicas o estenotípicas; de esta manera un centro de endemismo puede tan solo presentar un 5-10\% de especies endémicas y una subprovincia entre el 11-25\%, contrastando con las provincias (26$50 \%$ ), regiones $(51-75 \%$ ) o reinos $>75 \%$ (Salazar-Vallejo 2000, Ramírez-González 2005).

De las 62 especies de cangrejos ermitaños, 32 son generalistas según el análisis inverso, lo anterior demuestra que existe un alto grado de conectividad entre las agrupaciones. Sin embargo, no todas las especies generalistas se distribuyeron ampliamente, al contrario, la mayoría de las especies generalistas presentes al noreste, parecen estar ausentes al suroeste del Caribe colombiano, entre las ecorregiones de Golfo de Morrosquillo, Darien y San Andrés; mientras que las especies presentes al suroeste no están en las ecorregiones de Palomino y Guajira. En ambas situaciones se observó que los límites de distribución ocurrían entre las ecorregiones Tayrona, Magdalena y Archipielagos Coralinos, los cuales coinciden con los límites geográficos del grupo C, este se comporta como una zona de transición entre los componentes noreste y suroeste (Cuadro 4); este patrón coincide con lo observado en las especies presentes en el Caribe colombiano, con respecto al gran Caribe y el Atlántico; por lo que es probable que una transición similar a la observada a nivel local, también ocurra en el resto del Caribe; Duque-Caro (1980), afirma que el área definida en este trabajo como centro del Caribe colombiano, es el límite de dos 


\section{CUADRO 4}

Distribución de las especies generalistas en el análisis inverso

TABLE 4

Generalist species distribution in the inverse analysis of Kaandorp

\begin{tabular}{|c|c|c|c|}
\hline \multicolumn{4}{|c|}{ Distribución de las especies generalistas } \\
\hline Todo El Caribe Colombiano & NE - CEN & CEN y CGSM & SO - CEN \\
\hline Clibanarius tricolor & Agaricochirus alexandri & Clibanarius cubensis & Calcinus tibicen \\
\hline Clibanarius antillensis & Iridopagurus caribbensis & Clibanarius vittatus & Paguristes laticlavus \\
\hline Coenobita clypeatus & Iridopagurus violaceus & Paguristes puncticeps & Pagurus provenzanoi \\
\hline Dardanus fucosus & Paguristes oxiophthalmus & & \\
\hline Iridopagurus globulus & Pauristes sp. 3 & & \\
\hline Iridopagurus iris & Paguristes sp. 4 & & \\
\hline Iridopagurus reticulatus & Pagurus curaçaoensis & & \\
\hline Pagurus criniticornis & Pagurus longimanus & & \\
\hline Pagurus brevidactilus & Pagurus protuberocarpus & & \\
\hline Paragiopagurus pilimanus & Phimochirus holthuisi & & \\
\hline Petrochirus diogenes & Phimochirus operculatus & & \\
\hline \multirow[t]{3}{*}{ Xylopagurus anthonii } & Pylopagurus pattiae & & \\
\hline & Rhodochirus rosaceus & & \\
\hline & Tomopagurus wassi & & \\
\hline
\end{tabular}

De las 33 especies solo 13 se distribuyen ampliamente por el Caribe colombiano, mientras que las 20 restantes tienen como límite de distribución al sector CEN.

Of the 33 species only 13 are widespread in the Colombian Caribbean, whereas the remaining 20 have a boundary of distribution the CEN sector.

provincias tectónicas, una de origen oceánico y otra continental separadas por la cresta de la Beata. El área de origen continental corresponde a la Guajira y pudo estar conectada con el arco antillano, lo cual explica la relación de las especies del noreste del Caribe colombiano con las especies del Atlántico norte (IturraldeVinentt \& Macphee 1999); mientras que la región oceánica del suroeste, presentó cierto grado de aislamiento durante su evolución geomorfológica posterior al cierre del Istmo de Panamá (Díaz 1995, De Porta 2003); por lo cual, actualmente las especies de ermitaños representativos del sector suroeste presentan un alto grado de endemismo, respecto a las especies del resto del Caribe, y se encuentran exclusivamente en Colombia o bien desde Colombia hasta Honduras.

Diferencias entre los ensamblajes: $\mathrm{Si}$ bien según el $\mathrm{R}$ global, los grupos tienden a superponerse, el modelo, que conjuga el efecto de la profundidad, con los grupos NE, CEN y $\mathrm{SO}$ presenta mayor afinidad que algunos patrones propuestos anteriormente por otros autores, como las ecorregiones la sectorización NE-SO, donde el Río Magdalena actúa como barrera limítrofe entre las dos regiones (Merchán et al. 2009) (Cuadro 1). La baja correlación que se presenta entre los patrones de distribución de los ermitaños y las ecorregiones, puede atribuirse a que estas son áreas pequeñas, definidas por el predominio de algún tipo de ecosistema o característica oceanográfica o paisajística (IDEAM et al. 2007); sin embargo, por lo general los paisajes entre una ecorregión adyacente y otra tienden a ser similares, siempre que estos hagan parte de una misma unidad geomorfológica, por lo que sus límites no corresponden con las características que actúan como barreras geográficas para la distribución de los organismos. 
Los eventos oceanográficos y ecológicos han sido eficientes para identificar las diferencias entre el noreste y suroeste del Caribe colombiano y estos se han utilizado para discutir los patrones de distribución de las especies. Con base en esta información, el centro del Caribe, ha sido considerado como un área de transición y no como una unidad biogeográfica independiente, sin embargo, el análisis de similitud (Fig. 2) indica que los componentes faunísticos de Santa Marta y Cartagena, que se encuentran a cada extremo del río Magdalena, presentan mayor similitud entre sí, que la que podría tener la región de Cartagena y Archipiélagos Coralinos con el SO del Caribe, o la que podría tener la región de Santa Marta y Tayrona con el NE, por lo cual, es probable que el efecto del río sobre la zona no necesariamente represente un límite entre dos unidades biogeográficas y contrario a ello, el enriquecimiento debido a los efectos estacionales de la pluma del río Magdalena, pueda ser considerado como una condición característica del sector CEN, la cual, favorece el florecimiento de la fauna bentónica en las áreas adyacentes.

\section{AGRADECIMIENTOS}

Agradecemos al Instituto de Investigaciones Marinas y Costeras (INVEMAR) y al CECIMAR, de la Universidad Nacional de Colombia, Sede Caribe por suministrarnos el material recolectado durante los cruceros Macrofauna I, II y Macrofauna-Corpoguajira, asimismo por permitirnos hacer uso de las bases de datos, bibliografía e información geográfica. También agradecemos el apoyo recibido por los docentes de la Universidad Jorge Tadeo Lozano, en especial a Adolfo Sanjuan por su ayuda y acertados comentarios sobre los análisis estadísticos. Finalmente a Allan Valle y Sven Zea por su colaboración en la versión en inglés del resumen. INVEMAR: CTRB-1081 y CECIMAR: 362.

\section{RESUMEN}

Los cangrejos ermitaños son un grupo representativo de la fauna marina del Caribe colombiano, además, son importantes en el mantenimiento del equilibrio dinámico, en los ecosistemas por sus interacciones ecológicas y también por el impacto en la estabilidad de las redes tróficas. Sus patrones de distribución se identificaron mediante análisis multivariados de clasificación y ordenación espacial, se usaron registros históricos desde 1916 hasta el 2006. Los resultados indican diferencias por profundidad entre la fauna costera y la del talud continental y diferencias en la distribución latitudinal, en donde se encontraron tres grupos: Noreste, Centro y Suroeste, sustentados por diferencias en la composición faunística. Con base en los mapas de ecosistemas marinos de Colombia, se determinó que los principales factores que afectan su distribución son la influencia del talud Caribaná (profundidad), la temperatura de las masas de agua, las praderas de pastos marinos de la Guajira y las condiciones particulares de las ecorregiones Archipiélagos coralinos y Darién. Se identificó la distribución mundial de las especies colombianas y su afinidad geográfica, y se encontró mayor afinidad con el Atlántico norte y las Antillas que con el Atlántico sur y el Golfo de México, asimismo, las subprovincias geográficas que incluyen a Colombia son zonas de transición dentro de la subprovincias septentrionales y australes del Gran Caribe.

Palabras clave: cancrejos ermitaños, patrones de distribución, análisis multivariado, Caribe colombiano, distribución latitudinal, distribución por profundidad.

\section{REFERENCIAS}

Abele, L.G. \& W. Kim. 1986. An illustrated guide to the marine decapod crustaceans of Florida. Florida State University, Florida, EEUU.

Álvarez-León, R., L. Mendoza-Mazzeo \& G. Vernette. 2003. Factores de formación de las lagunas costeras del suroeste del Caribe colombiano. Acta Cient. Venez. 54: 180-188.

Andrade, C., F. Arias \& I. Thomas. 1988. Estudio temporal de los sólidos en suspensión entre la desembocadura del río Magdalena y el Canal del Dique, Mar Caribe Colombia.VI Sem. Nac. Ciencias del Mar. Universidad Jorge Tadeo Lozano y la Comisión Colombiana de Oceanografía, Bogotá, Colombia.

Andrade, C., E. Barton \& C. Mooers. 2003. Evidence for a eastward flow along the Central and South American Caribean Coast. J. Geo. Res. 108: 3185-3196. 
Ardila, N., G.R. Navas \& J. Reyes. 2002. Libro rojo de los invertebrados marinos de Colombia. La serie Libros rojos de especies amenazadas de Colombia. INVEMAR, Ministerio de Medio Ambiente, Bogotá, Colombia.

Bermúdez, A. 2000. Diversidad y distribución de los crustáceos decápodos de la franja superior del talud continental (300-500m de profundidad) en la parte norte del Caribe colombiano. Trabajo de grado en Biólogía, Universidad Nacional de Colombia, Bogotá, Colombia.

Bermúdez, A. 2005. Caracterización morfológica de las poblaciones de Agononida longipes y Munida flinti (Crustacea: Decapoda: Galatheidae) en el Mar Caribe colombiano. Tesis Maestría, Universidad Nacional de Colombia, Bogotá, Colombia.

Bermúdez, A. 2007. Análisis poblacional de las especies americanas Munida flinti, Agononida longipes y Munidopsis riveroi (Crustacea, Decapoda, Anomura: Galatheidae). Inaugural-Dissertation Doctor en Ciencias Naturales (Dr. rer. nat.), Justus-LiebigUniversität Gießen, Gießen, Alemania.

Bermúdez, A., G.R. Navas \& N.H. Campos. 2008. Populationsstruktur und genetische Isolierung von drei Springkrebs-Arten. Der Einfluss der ozeanographischen Merkmale und der geographischen Distanz zwischen den Populationen entlang der karibischen Küste von Kolumbien. VDM Verlag, Saarbrücken, Alemania.

Bernal, G., G. Poveda \& C. Andrade. 2006. Patrones de variabilidad de las temperaturas superficiales del mar en la costa Caribe Colombiana. Rev. Acad. Col. Cienc. 30: 195-208

Bertness, M.D. 1981. Shell utilization, predation pressure, and thermal stress in panamanian hermit crabs: An interoceanic comparison. J. Exp. Mar. Biol. Ecol. 64: $159-187$.

Blanco, J.A. 1988. Las variaciones ambientales estacionales en las aguas costeras y su importancia para la pesca en la región de Santa Marta Caribe colombiano. Tesis Maestría, Universidad Nacional de Colombia, Santa Marta, Colombia.

Campos, N.H. 1978. Los cangrejos ermitaños de la bahía de Cartagena familia Paguridae y su biología. Trabajo de grado en Biólogía Marina, Universidad Jorge Tadeo Lozano, Santa Marta, Colombia.

Campos, N. \& H. Sánchez. 1995. Los cangrejos ermitaños del género Paguristes Dana (Anomura: Diogenidae) de la costa norte colombiana con la descripción de dos nuevas especies. Caldasia 17: 569-586.

Campos, N.H., G.R. Navas, A. Bermúdez \& N. Cruz. 2005. Los crustáceos decápodos de la franja superior del talud continental (300-500m) del Mar Caribe colombiano. Monografías de fauna de Colombia. Universidad Nacional de Colombia, Instituto de Ciencias Naturales, Bogotá, Colombia.

Clarke, K. \& M. Ainsworth. 1993. A method of linking multivariate community structure to environmental variables. Marine Ecol. Progr. Ser. 92: 205-219.

Clarke, K.R. \& R.N. Gorley. 2006. PRIMER v6: User Manual/Tutorial. PRIMER-E: Plymouth, Inglaterra.

Cowen, R.K., C.B. Paris \& A. Srinivasan. 2006. Scaling of Connectivity in Marine Populations. Science 311: 522-527.

Criales-Hernández, M.I., C.B. García \& M. Wolff. 2003. Flujos de biomasa y estructura de un ecosistema de surgencia tropical en La Guajira, Caribe colombiano. Int. J. Trop. Biol. 54: 1257-1282.

Cruz, N. 2001. Caracterización de los crustáceos decápodos de la franja superior del talud continental (300$500 \mathrm{~m}$ de profundidad) en la parte sur del Mar Caribe colombiano. Trabajo de grado en Biología, Universidad Nacional de Colombia. Bogotá, Colombia.

De Porta, J. 2003. La formación del istmo de Panamá. Su incidencia en Colombia. Rev. Acad. Col. Cienc. Exact. Fis. Nat. 27: 191-216.

Díaz, J.M. 1995. Zoogeography of marine gastropods in the southern Caribbean: a new look at provinciality. Caribb. J. Sci. 31: 104-121.

Díaz, J.M. \& A. Acero. 2003. Marine biodiversity in Colombia: Achievements status of the knowledge and challengers. Gayana 67: 261-274.

Díaz, J.M. \& D.I. Gómez-López. 2000. Programa nacional de investigación en biodiversidad marina y costera. INVEMAR, FONADE, MMA, Santa Marta, Colombia.

Díaz-Pulido, G. \& M. Díaz-Ruíz. 2003. Diversity of benthic marine algae of the Colombian Atlantic. Biota Colombiana 4: 203-246.

Duque-Caro, H. 1980. Geotectónica y evolución de la región noroccidental colombiana. Boletín geológico, Ingeominas, Bogotá, Colombia.

García-Gómez, J. 1983. Revision of Iridopagurus (Crustacea: Decapoda: Paguridae) with the descriptions of 
new species from American waters. Bull. Mar. Sci. 33: $10-54$.

Gherardi, F. 1990. Competition and coexistence in two Mediterranean hermit crabs, Calcinus ornatus (Roux) and Clibanarius erythropus (Latreille) (Decapoda, Anomura). J. Exp. Mar. Biol. Ecol. 143: 221-238.

Hendrix, M.E. 2003. Geographic and bathymetric distribution of species of Munidopsis (Crustacea: Decapoda: Galathaeidae) in the SE Gulf of California, Mexico, p. 21- 30. In M.E. Hendrickx (ed.). Contribuciones al Estudio de los Crustáceos del Pacífico Este 2. Instituto de Ciencias del Mar y Limnología, UNAM, México D.F., México.

IDEAM, IGAC, IAvH, INVEMAR, I. Sinchi \& IIAP. 2007. Ecosistemas continentales, costeros y marinos de Colombia. Instituto de Hidrología, Meteorología y Estudios Ambientales, Instituto Geografico Agustin Codazzi, Instituto de Investigación de Recursos Biologicos Alexander von Humboldt, Instituto de Investigaciones Ambientales del Pacifico Jhon von Neumann, Instituto de Investigaciones Marinas y Costeras José Benito Vives De Andreis e Instituto Amazónico de Investigaciones Científicas Sinchi. Bogotá, Colombia.

Iturralde-Vinent, M.A.\& R.D.E. Macphee. 1999. Caribbean paleogeography. Bull. Am. Mus. Nat. Hist. 238: 1-95.

Kaandorp, J. 1986. Rocky substrate communities of the infralitoral fringe of the boundaries coast, NW France: A quantitative survey. Mar. Biol. 92: 22-256.

Latreille, P.A.1802. Histoire naturelle, générale et perticuliére, des crustaces et des insectes. F. Dufart, París, Francia.

Lemaitre, R. 1989. Revision of the genus Parapagurus (Anomura: Paguroidea: Parapaguridae), including redescriptions of the Western Atlantic species. Zoologische Verhandel. 253: 1-106.

Lemaitre, R. \& N.H. Campos. 1993. Two new hermit crabs (crustacea: decapoda: Paguridea) from the Caribean sea. Proc. Biol. Soc. Wash. 106: 554-565.

Lemaitre, R. \& P.A. McLaughlin. 1996. Revision of Pylopagurus and Tomopagurus (Crustacea: Decapoda: Paguridae), with the descriptions of new genera and species. Part V. Anisopagurus Mclaughlin, and Protoniopagurus new genus. Bull. Mar. Sci. 59: 89-141.

Lemaitre, R., P.A. McLaughlin \& J. García-Gómez. 1982. The Provenzanoi group of hermit crabs (Crustacea: Decapoda: Paguridae) in the western Atlantic. Part IV: A review of the group, with notes on variations and abnormalities. Bull. Mar. Sci. 32: 670-701.
Mantellato, F.L.M. \& L.C.C. Dominiciano. 2002. Pattern of shell utilization by the hermit crab Paguristes tortugae (Diogenidae) from Anchieta Island, Southern Brazil. Sci. Mar. 66: 265-272.

Martínez, B. 2010. Biología y patrones de distribución de los cangrejos ermitaños (Crustacea: Decapoda: Anomura: Paguridea) en el mar Caribe colombiano. Tesis de grado en Biología Marina, Universidad de Bogotá Jorge Tadeo Lozano, Bogotá D.C., Colombia.

McLaughlin, P.A. 1975. Biological results of the University of Miami deep-sea expeditions. 114. On the identity of Pagurus brevidactylus (Stimpson) (Decapoda: Paguridae), with the description of a new species of Pagurus from de western Atlantic. Bul. Mar. Sci. 25: $359-376$.

McLaughlin, P.A. \& J. Haig. 1989. On the status of Pylopaguropsis zebra (Henderson) P. magnimanus (Henderson), and Galapagurus teevanus Bone, with descriptions of seven new species of Pylopaguropsis (Crustacea; Anomura; Paguridae). Micronesica 2: $123-171$.

McLaughlin, P.A. \& R. Lemaitre. 2001. Revision of Pylopagurus and Tomopagurus (Crustacea: Decapoda: Paguridae), with the descriptions of the genera and species. Part VI. Pylopagurus A. Milne-Edwards \& Bouvier, 1891, Hagia Mclaughlin, 1981, and Pylopaguridium, a new genus. Proc. Biol. Soc. Wash. 114: 444-483.

McLaughlin, P.A. \& A. Provenzano Jr. 1974a. Hermit crabs of the genus Paguristes (Crustacea: Decapoda: Diogenidae) from Western Atlantic. Part. II. Descriptions of six new species. Bul. Mar. Sci. 24: 885-938.

McLaughlin, P.A. \& A. Provenzano Jr. 1974b. Hermit crabs of the genus Paguristes (Crustacea: Decapoda: Diogenidae) from Western Atlantic. Part. I. The Paguristes tortugae complex, with notes on variation. Bull. Mar. Sci. 24: 165-233.

Mejía, M. 1989. Caribe colombiano: Clima y relieve con referencia al uso de la tierra cuadernos de geografía. IGAC 1: 13-29.

Merchán, A., N.H. Campos, A. Franco \& A. Bermúdez. 2009. Distribución y datos biológicos de los cangrejos ermitaños (Decapoda: Anomura) del mar Caribe colombiano colectados por la expedición Macrofauna II. Bol. Invest. Mar. Cost. 28: 121-142.

Navas, G.R, A. Bermúdez, N. Crúz \& N.H. Campos. 2003. Galatéideos (Crustacea, Decapoda, Anomura, Galatheidae) del Caribe colombiano, incluyendo 12 primeros registros. Bol. Invest. Mar. Cost. 32: 183-218. 
Pearce, A.S. 1916. An account of the crustacean collected by the Walker Expedition to Santa Marta, Colombia. Proc. U.S. Nat. Mus. Wash. 49: 21-23.

Pequegnat, L.H. \& W.E. Pequegnat. 1970. Deep sea anomurans of superfamily Galatheoidea with descriptions of three new species, p. 125-170. In W.E. Pequegnat \& F.A. Chace Jr. (eds.). Contributions on the Biology of the Gulf of México. Texas A\&M Univ. Oceanogr. Studies, Texas, EEUU.

Posada, B.O. \& D.M. Rozo. 2002. Marco geográfico, p. 16-31. In INVEMAR (ed.). Informe del estado de los ambientes marinos y costeros en Colombia. INVEMAR, Santa Marta, Colombia.

Provenzano Jr., A.J. 1965. Two West Indian hermit crabs of the genus Paguristes (Crustacea, Diogenidae). Bull. Mar. Sci. 15: 726-736.

Ramírez-González, A. 2005. Ecología aplicada. Diseño y análisis estadístico. Colección estudios de ecología. Universidad de Bogotá Jorge Tadeo Lozano, Bogotá D.C., Colombia.

Rex, M.A. 1977. Zonation in deep-sea Gastropods: The importance of the biological interaction to rates interactions, p. 521-531. In B.F. Keegan, T.O. Ceidgh \& P.J.S. Boaden (eds.). Biology of benthic organisms. Pergamon, Londres, Inglaterra.

Sánchez, H. \& N.H. Campos. 1978. Cangrejos ermitaños (Crustacea, Decapoda, Anomura, Paguridae) de la costa norte Colombiana. An. Inst. Inv. Mar. Punta Betín. Santa Marta (Colombia) 10: 15-72.

Santodomingo, N., M. Rueda, J.M. Díaz, N.E. Ardila, D.P Báez, G. Borrero, A. Gracia, L.S. Mejía, L.M. Mejía \& J. Reyes. 2004. Estado de la diversidad de especies marinas en Colombia, p. 225-257. In INVEMAR (ed.). Informe del Estado de los Ambientes Marinos y Costeros en Colombia: Año 2003. Servigráficas, Medellín, Colombia.

Salazar-Vallejo, S.I. 2000. Biogeografía marina del Gran Caribe. Interciencia 25: 1-12.

Sanders, H.L. \& R.R. Hessler. 1969. Ecology of the deep sea benthos. Science 163: 1419-1424.

Steer, R., F. Arias, A. Ramos, P. Aguirre, P. Sierra \& D. Alonso. 1997. Documento preliminar de políticas de ordenamiento ambiental de las zonas costeras colombianas. Documento de consultoría. Ministerio del Medio Ambiente, Bogotá, Colombia.

Tomczak, M. 1999. Global water mass analysis, a symposium of IAPSO at the IUGG General Assembly. International WOCE Newsletter 36: 10-11.

Wass, M.L. 1963. New species of hermit crabs (Decapoda: Paguridae) from the Western Atlantic. Crustaceana 6: 133-157.

Wenner, E.L. \& D.F. Boesch. 1979. Distribution patterns of epibenthic Decapod, Crustacea along the shelf-slope coenocline. Middle Atlantic Bight, U.S.A. Bull. Biol. Soc. Wash. 3: 106-133.

Williams, J.D. \& J.J. McDermott. 2004. Hermit crab biocoenoses: a worldwide review of the diversity and natural history of hermit crab associates. J. Exp. Mar. Biol. Ecol. 305: 1-128.

WoRMS. 2010. World Research Marine Species. Aukland University, Aukland, EEUU. (Consultado: 17 de Agosto de 2010, www.marinespecies.org/ downloads.php). 